\title{
An Atmospheric Variability Model for Venus Aerobraking Missions
}

\author{
Robert H. Tolson ${ }^{\star}$, Jill L. H. Prince ${ }^{\dagger}$ and Alexander A. Konopliv
}

\begin{abstract}
Aerobraking has proven to be an enabling technology for planetary missions to Mars and has been proposed to enable low cost missions to Venus. Aerobraking saves a significant amount of propulsion fuel mass by exploiting atmospheric drag to reduce the eccentricity of the initial orbit. The solar arrays have been used as the primary drag surface and only minor modifications have been made in the vehicle design to accommodate the relatively modest aerothermal loads. However, if atmospheric density is highly variable from orbit to orbit, the mission must either accept higher aerothermal risk, a slower pace for aerobraking, or a tighter corridor likely with increased propulsive cost. Hence, knowledge of atmospheric variability is of great interest for the design of aerobraking missions. The first planetary aerobraking was at Venus during the Magellan mission. After the primary Magellan science mission was completed, aerobraking was used to provide a more circular orbit to enhance gravity field recovery. Magellan aerobraking took place between local solar times of 1100 and 1800 hrs, and it was found that the Venusian atmospheric density during the aerobraking phase had less than $10 \% 1 \sigma$ orbit to orbit variability. On the other hand, at some latitudes and seasons, Martian variability can be as high as $40 \% 1 \sigma$. From both the MGN and PVO mission it was known that the atmosphere, above aerobraking altitudes, showed greater variability at night, but this variability was never quantified in a systematic manner. This paper proposes a model for atmospheric variability that can be used for aerobraking mission design until more complete data sets become available.
\end{abstract}

\section{Nomenclature}

$\begin{array}{ll}\text { AB } & \text { Aerobraking } \\ \mathrm{H}_{\mathrm{s}} & \text { Density scale height, km } \\ \text { IMU } & \text { Inertial Measurement Unit } \\ \text { LST } & \text { Local Solar Time of Sun relative to satellite orbit periapsis } \\ \text { MGN } & \text { Magellan } \\ \text { MGS } & \text { Mars Global Surveyor } \\ \text { MRO } & \text { Mars Reconnaissance Orbiter } \\ \text { ODY } & \text { Mars Odyssey } \\ \text { PVO } & \text { Pioneer Venus Orbiter } \\ \text { SZA } & \text { Solar Zenith Angle of Sun relative to satellite orbit periapsis } \\ \text { T } & \text { atmospheric temperature } \\ \text { VIRA } & \text { Venus International Reference Atmosphere } \\ & \\ \text { a, } \mathrm{a}_{\mathrm{v}} & \text { satellite orbit and Venus orbit semi-major axes, km } \\ \mathrm{e} & \text { orbit eccentricity or base of natural logarithms } \\ \mathrm{g} & \text { local gravitational acceleration } \\ \mathrm{h}, \mathrm{h}_{\mathrm{o}} & \text { planetodetic altitude \& reference altitude, km } \\ \mathrm{k} & \text { Boltzmann constant } \\ \mathrm{m} & \text { mean molecular weight }\end{array}$

* Langley Professor Emeritus, NIA, 100 Exploration Way, Hampton, VA 23666

$\uparrow$ Head, Structural and Thermal Systems Branch, NASA LaRC, MS 489, Hampton, VA 23681

\# Solar Systems Dynamics Group, JPL, 301-121, Pasadena, CA 91109 


$$
\begin{array}{ll}
\phi & \text { latitude of Sun in orbit plane system } \\
\mu_{\mathrm{v}}, \mu_{\mathrm{s}} & \text { Venus and Sun gravitational constants, } \mathrm{km}^{3} / \mathrm{s}^{2} \\
\rho & \text { atmospheric density, } \mathrm{kg} / \mathrm{km}^{3} \\
\theta & \text { longitude of Sun in orbit plane system }
\end{array}
$$

\section{Introduction}

The first planetary aerobraking (AB) mission, Venus Magellan (MGN), took 70 days and over 700 passes through the atmosphere for the purpose of enhancing the scientific return of the extended mission ${ }^{1}$. After the primary science mission, aerobraking was performed to reduce the orbital eccentricity thereby lowering apoapsis altitude and improving the resolution of gravity mapping at high latitudes. Based on the successful Magellan experience, $\mathrm{AB}$ became an enabling technology for recent Mars orbiting missions. These missions had AB operational phases that took about 850 orbits for Mars Global Surveyor (MGS), 77 days and 325 orbits for Mars Odyssey (ODY), and 145 days and 420 orbits for Mars Reconnaissance Orbiter (MRO). MGS was anomalistically long due to a broken solar array which constrained the maximum dynamic pressure during an $\mathrm{AB}$ pass. These missions used the solar arrays as the primary drag area and consequently, except for MGS, the temperature of the solar arrays was the limiting atmosphere dependent factor in designing the AB corridor, ${ }^{1}$ although other subsystems had to also be considered.

Since aerodynamic heating is generally the critical consideration, aerobraking missions typically have a heat flux or total heat load corridor. This corridor is generally mapped into an atmospheric density corridor that defines the range of permissible periapsis altitudes. During early operations, called the walk-in phase, propulsive maneuvers are used to incrementally lower periapsis altitude to sense the proper altitude at which to initiate aerobraking. Hence, precise knowledge of atmospheric density vs. altitude is not required. Once 'main phase' aerobraking begins, altitude adjustment maneuvers are utilized to maintain periapsis within the density corridor. The variation of density with altitude, as specified by an engineering atmospheric model or determined by flight data, is used to design corridor control maneuvers. The major risk to this approach is the unmodeled orbit to orbit variations in density which are generally not captured by current engineering models. For Mars, orbit to orbit variability can be more than a factor of two primarily due to global scale, longitude dependent density wave structure. ${ }^{2}$ Consequently, Mars aerobraking vehicles have been designed with at least a factor of 2 safety margin. Due to the limited experience with orbiting missions at aerobraking altitudes, there is a dearth of data on which to quantify such variability at Venus. This paper will attempt to use the limited Magellan data during aerobraking and the MGN and Pioneer Venus Orbiter (PVO) data at higher altitudes to quantify orbit to orbit variability.

\section{Data Description}

This study is based on atmospheric density estimates generated while determining a gravity model for Venus ${ }^{3}$ from MGN and PVO radio tracking data. To accommodate atmospheric drag in the OD process, atmospheric density was estimated at the reference altitude of $140 \mathrm{~km}$. For most orbits of MGN and PVO periapsis was well above 140 $\mathrm{km}$, so a version of the VIRA model was used to relate atmospheric density at the reference altitude to the density in the vicinity of periapsis. As that study focused on the gravity field, there was no effort to recover precise atmosphere density and it is to be noted that a constant drag coefficient of 2.2 was used for the entire study and relatively simple models of vehicle geometry and attitude. Below the homopause, $\mathrm{CO}_{2}$ is the dominant species. During the Venusian day, atomic oxygen dominates between about $160 \mathrm{~km}$ to $200 \mathrm{~km}$ and at night above about $140 \mathrm{~km}$. Since the drag coefficient is affected by such changes ${ }^{4}$ and for this and other reasons, not much attention will be focused on differences between high altitude density results and VIRA or other atmospheric models. However, such potential aliasing in the drag coefficient will not likely significantly influence the orbit to orbit fractional density variability of interest here. A final note is that during periods of interest, for both missions, the latitude of periapsis latitudes was limited between $10 \mathrm{~N}$ and $20 \mathrm{~N}$.

Figure 1 shows the altitude and local solar time (LST) during the phases of both mission which were sensitive to atmospheric drag. The MGN mission was divided into 6 cycles with each providing about 1 Venus day of coverage. The first three cycles were devoted to synthetic aperture radar mapping of the surface. The periapsis altitude was lowered during cycle 4 to enhance gravity field recovery. Following cycle 4 , the aerobraking (AB) phase ${ }^{5}$ provided a much more circular orbit to enhance gravity field recovery in the polar regions. MGN aerobraking took place over a range of LST from 1000 to $1800 \mathrm{hrs}$ and over an altitude range from 135 to $142 \mathrm{~km}$. These data provide an excellent opportunity to recover orbit to orbit variability for daytime aerobraking. It is an open question as to the orbit to orbit 
variability during nighttime at Venus at $\mathrm{AB}$ altitudes. Since the atmosphere contracts significantly at night, $\mathrm{AB}$ at night would likely occur about $10 \mathrm{~km}$ lower in altitude than daytime AB. As seen from Figure 1, there are few night side data below $150 \mathrm{~km}$, and none near 130 where night side AB would likely occur. Further, the accuracy of recovering density from orbit changes decreases rapidly with periapsis altitude as density decreases expotentially and drag has a less significant influence on orbit changes. As altitude increases the orbit determination uncertainty will contribute a larger fraction to the observed variability and must be included in the analysis. Fortunately, error estimates were provided along with the data.
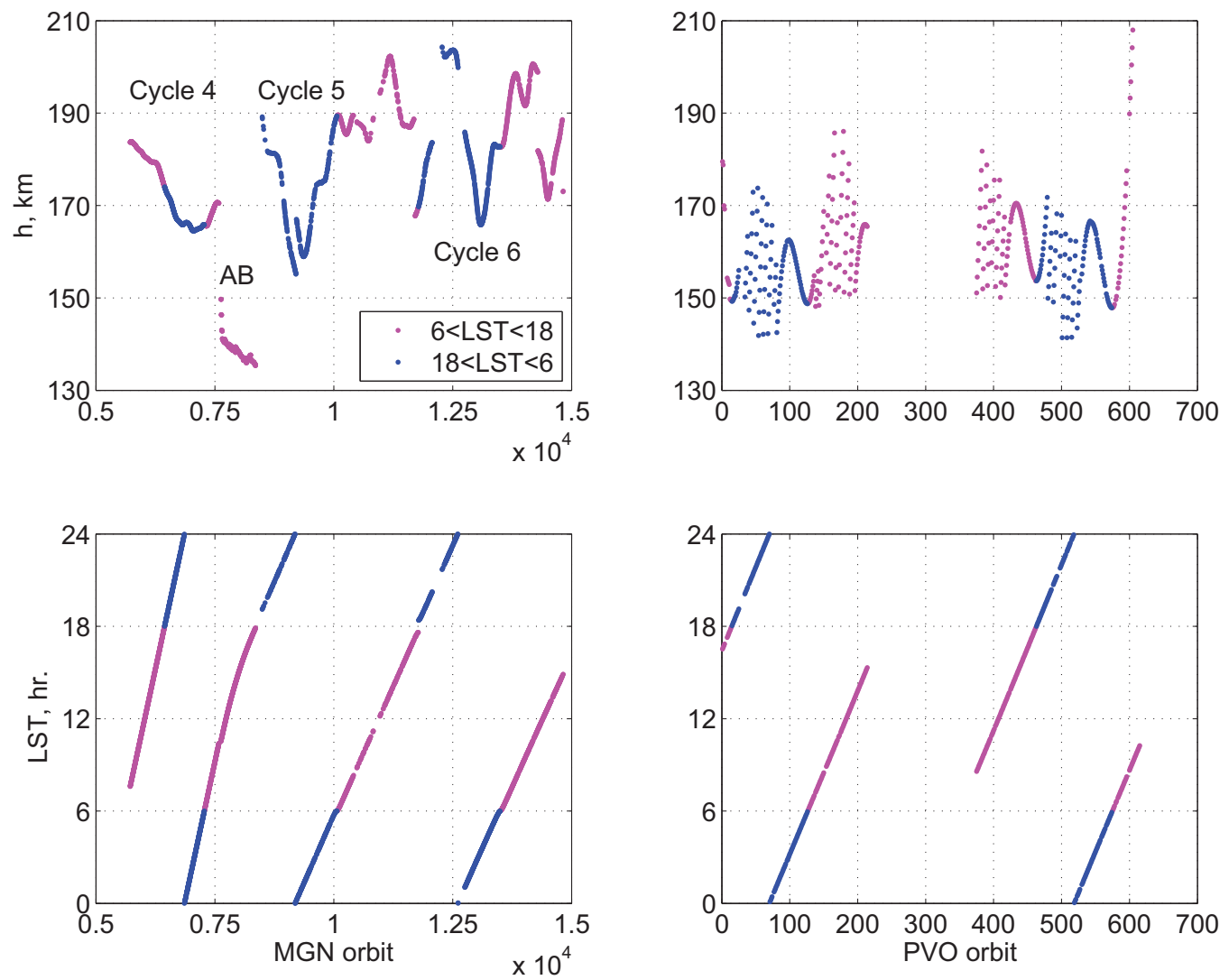

Figure 1. Magellan and Pioneer Venus Orbiter altitude and local solar time during mission phases at drag sensitive altitudes.

The PVO mission provides data from a 24 hour orbit for two Venus days with the lowest periapsis on the night side, but only down to about $140 \mathrm{~km}$ altitude. PVO had an orbit inclination of about $106^{\circ}$ and periapsis latitude varied from about $13^{\circ}$ to $17^{\circ}$. The MGN orbit inclination was about $86^{\circ}$ and latitude varied from about $11^{\circ}$ to $45^{\circ}$, with the higher latitudes occurring at the end of cycle 6 when the periapsis altitude was above $170 \mathrm{~km}$. The MGN orbital period was never longer than $3.5 \mathrm{hrs}$, so the two missions are sampling the atmosphere with different temporal frequency. Both periods are very short compared to the length of the Venusian day, but it is unknown how these periods compare to the atmospheric natural variability temporal spectrum. Venus has a relatively small non-central gravity field that produces orbit perturbations that are small compared to satellites at more rapidly rotating planets like Mars and Earth. On the other hand, the solar gravitational effects are larger for the orbits with longer periods. Note that the PVO periapsis altitude frequently changes by at least $5 \mathrm{~km}$ during one orbit for certain Sun-orbit configurations. This change is about one density scale height and would produce a $270 \%$ change in density. Aerobraking with such large changes will likely take an orbit trim maneuver every orbit to stay in the AB corridor. On the other hand, it will be seen that the MGN periapsis altitude changes by much less than a kilometer due to non-central gravitation effects. These differences imply that the aerobraking corridor strategy will depend strongly on the orbital period. Of course, periapsis altitude can be predicted with sufficient accuracy for aerobraking using current gravity models. ${ }^{3}$ 
Figure 2 shows the orbit to orbit change in periapsis altitude due to solar gravity for a 24 hour orbital period Venusian satellite with periapsis altitude of $140 \mathrm{~km}$. Results are from the variation of parameters approach to third body perturbation given by ${ }^{6}$

$$
\Delta r_{p}=7.5 \pi\left[\frac{\mu_{s}}{\mu_{v}}\right] e \sqrt{1-e^{2}}\left[\frac{a}{a_{v}}\right]^{3} \sin 2 \theta \cos ^{2} \phi
$$

Orbit semi-major axis (a) and eccentricity (e) can of course be determined from the two AB relevant parameters: orbital period and periapsis radius. Results were compared with precision numerical simulation and differences were negligible for orbital periods of interest to $\mathrm{AB}$ missions. The location of the Sun is defined in the satellite orbit plane coordinate system. The origin is the center of mass of Venus and the orbit plane is the reference plane, i.e. the $\mathrm{z}$-axis is normal to the orbit plane, and periapsis lies on the $+\mathrm{x}$-axis. In this system the location of the Sun is provide by longitude $(\theta)$ measured in the orbit plane from the $\mathrm{x}$-axis and latitude or elevation $(\phi)$. From equation 1 , the locations where $\Delta r_{p}=0$ correspond to times when the Sun is either in the $x-z$ plane or $y-z$ plane, i.e. $\bmod (\theta, \pi / 2)=0$. Since $\cos S Z A=\cos \theta \cos \phi$, sunrise and sunset are included in this set. The largest changes occur at extremals of $\sin 2 \theta \cos ^{2} \phi$ and depend on orbit geometry. For a 24 hour orbit with a periapsis altitude of $140 \mathrm{~km}$, the maximum change from one orbit to the next is $8.3 \mathrm{~km}$ and for a 5 hour orbit the maximum change is about $0.1 \mathrm{~km}$. Because of the possibility of large periapsis altitude orbit-to-orbit variations, aerobraking considerations, such as propulsive requirements and aerobraking risk, may play a larger role in post-VOI orbit design that in post-MOI orbit design.

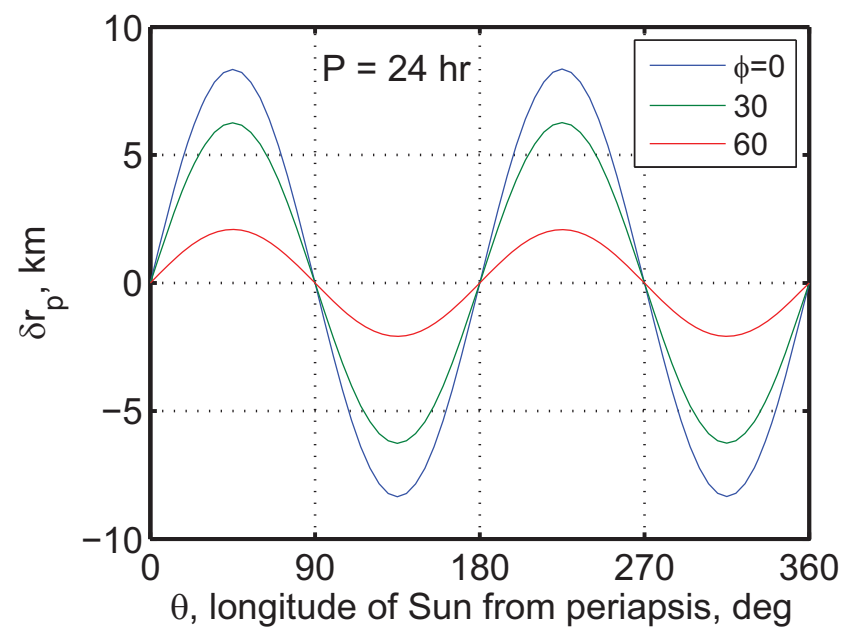

Figure 2. Solar third body gravity perturbation on orbit to orbit periapsis altitude for 24 hour orbit at Venus.

\section{Thermospheric Variability during Magellan Aerobraking and Cycle 4}

Figure 3 show MGN altitude, density, orbital period and LST for the AB phase. The "walk-in" phase is from 150 $\mathrm{km}$ to $141 \mathrm{~km}$ and then "main phase" aerobraking begins. The orbit to orbit variability in periapsis altitude due to non-propulsive forces is less than $100 \mathrm{~m}$ and no more than $3 \%$ of a density scale height. This is very small compared to those seen during Mars AB missions where they can be as large as $2 \mathrm{~km}$ or about $30 \%$ of the density scale height. The maneuvers to maintain periapsis altitude within the aerobraking corridor are clearly evident and are generally in the 0.5 to $1 \mathrm{~km}$ range.

Traditional aerobraking operations requires an estimate of the atmospheric density profile that describes, as a minimum, both the density and density scale height. The simplest model is usually derived by assuming an isothermal temperature profile and hydrostatic equilibrium, resulting in

$$
\rho(h)=\rho\left(h_{o}\right) e^{\left(\frac{h-h_{o}}{H_{s}}\right)}
$$

where $\mathrm{h}$ is the altitude, $\mathrm{h}_{\mathrm{o}}$ is the reference altitude (usually periapsis) and $\mathrm{H}_{\mathrm{s}}$ is the density scale height. Under these assumptions, $H_{s}=k T / m g$ and for both MGN and PVO the VIRA profiles of $H_{s}$ were used during operations and $\rho\left(h_{0}\right)$ 
was determined from radio tracking. After MGN, all Mars aerobraking missions derived both of these quantities from IMU accelerometer data taken during the aerobraking phase of the orbit. ${ }^{7}$ More complicated models have been used during operations and proposed for autonomous aerobraking applications ${ }^{8}$. The variability of the atmosphere was defined in terms of the deviation of the 'to be measured' atmosphere from projections based on the constant scale height density model above. Since there was no accelerometers on either MGN or PVO, the densities at periapsis will be used to derive the variability by studying the deviations from the exponential model over a number of adjacent orbits. To this end, the MGN AB phase was divided into a number of short arcs: "walk-in" and main phase orbits between periapsis raise or lower maneuvers. These arcs are easily identified in the first panel of Figure 3 . In the fourth panel, it is clearly seen that atmospheric density, during the 39 orbit walk-in from 150 to $141 \mathrm{~km}$, is approximately exponential while that trend is not so obvious for the rest of the AB phase.
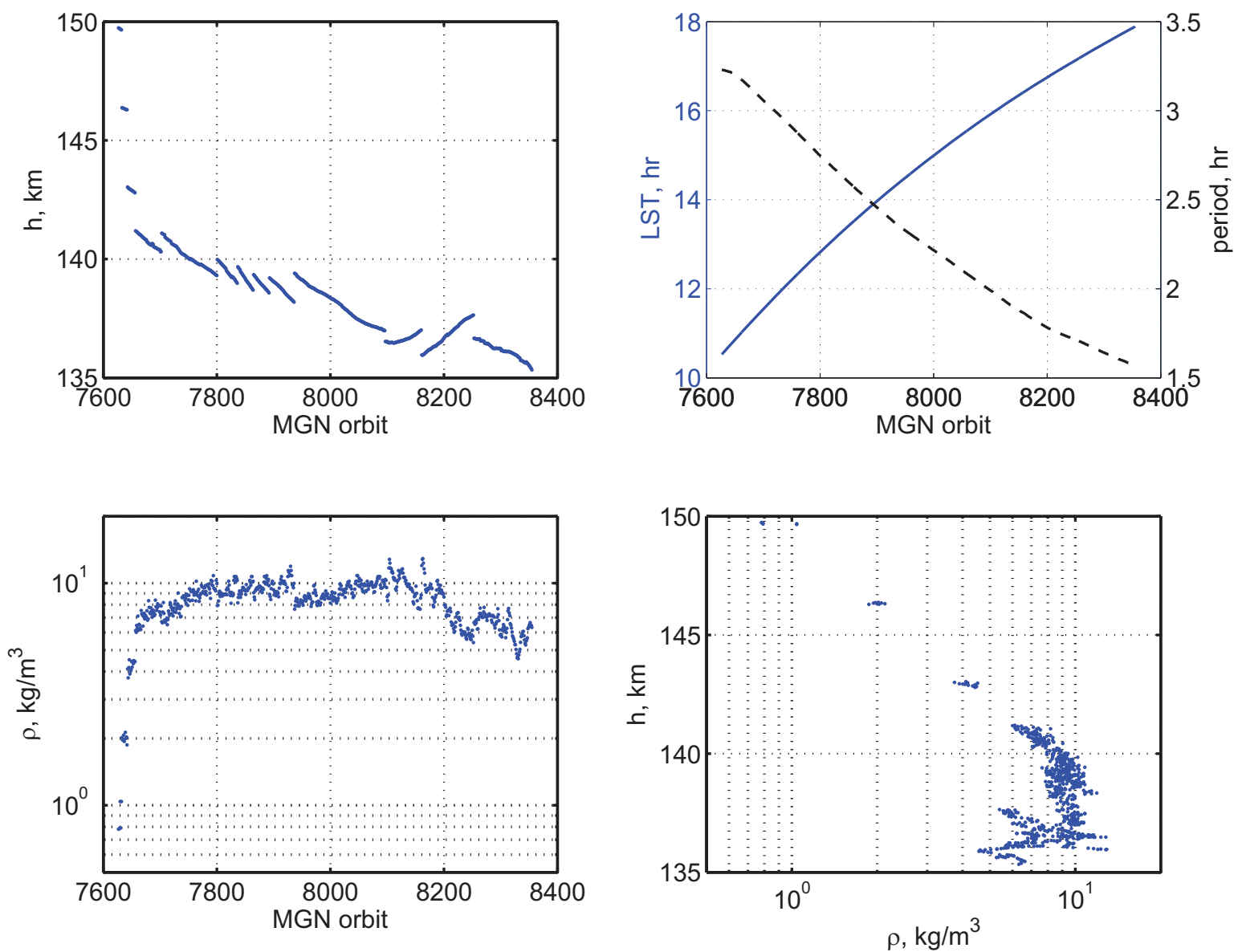

Figure 3. Characteristics of Magellan aerobraking altitude, LST and density.

The results of analyzing each arc are shown in Figure 4. The red line provides the exponential model for that arc obtained by a linear least squares fit to solve for $\log \left(\rho\left(\mathrm{h}_{\mathrm{o}}\right)\right)$ and $-1 / \mathrm{H}_{\mathrm{s}}$. The one sigma density deviation $(\sigma)$ from this model is provided along with the derived density scale height with a minimum $\mathrm{H}_{\mathrm{s}}$ set at $3.9 \mathrm{~km}$. The local solar time span for each arc is shown along with the number of orbits in the solution. The walk-in phase, over $15 \mathrm{~km}$ and nearly 4 scale heights, only shows a 7\% standard deviation. The other arcs cover an altitude range that is smaller than the derived scale height. Generally this would lead to a poorly determined scale height, but for most of these arcs, the deviation from the model is so small that the $\mathrm{H}_{\mathrm{s}}$ estimates seem reasonable and consistent between adjacent arcs. The values also generally agree with the VIRA model values which increase throughout the morning, reaches a peak or around $6 \mathrm{~km}$ between 1400 and 1500 hours, and decreases to around $4 \mathrm{~km}$ at sunset. However, the $\mathrm{H}_{\mathrm{s}}$ values after 1400 hrs. are much smaller than VIRA values. Further, one hour before sunset, the concept of a density scale height looses all meaning as a strong LST density gradient appears to dominate over the altitudinal variation. The $\sigma$ in this panel is the standard deviation about the mean of the density measurements. This can be seen vividly in Figure 3 
where altitude is decreasing over the last arc but density is also generally decreasing. The LST density gradient also biases the $\mathrm{H}_{\mathrm{s}}$ obtained by the exponential fit at earlier hours. If altitude is increasing, the apparent density increases faster than the altitudinal effect only, leading to an underestimation of $\mathrm{H}_{\mathrm{s}}$. Conversely, if altitude is decreasing, $\mathrm{H}_{\mathrm{s}}$ is overestimated. Similar, but less drastic, latitudinal gradients were seen during Mars aerobraking as periapsis precessed toward or away from a pole. ${ }^{7}$ Finally, the $1 \sigma$ deviations are plotted against LST in the bottom right panel with the conclusion being that deviations from the simple exponential model can be expected to increase rapidly as one approaches the terminator. and the derived $\mathrm{H}_{\mathrm{S}}$ is of questionable value in estimating density variation with altitude. Until data become available to estimate $\mathrm{H}_{\mathrm{s}}$ from a single periapsis pass, it is not known if this conclusion will apply when traditional accelerometer data are available. The conclusion will likely depend on the LST or SZA range covered during the $\mathrm{AB}$ pass.
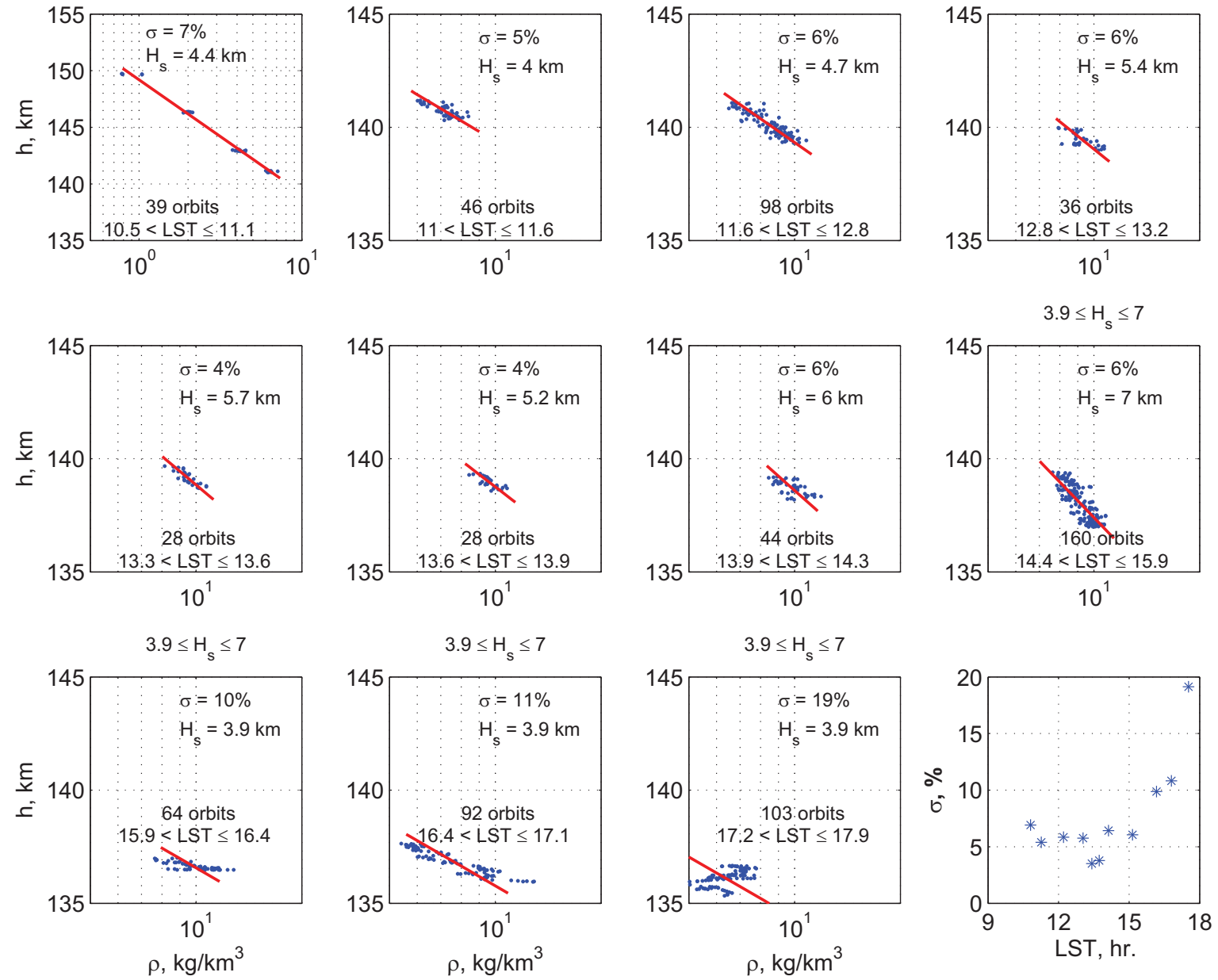

Figure 4. Magellan aerobraking variability based on deviation from an exponential model. 
From Figure 3 it is seen that the arcs are densely packed with orbits that have very small altitude differences between adjacent orbits. In this situation, one might ask if an altitudinal model is even required to predict the density at the next orbit. Closer study, provided in Figure 5, shows that there are isolated periapsis altitude changes of order 50 meters. These could be due to local gravity anomalies, changes in the batch of orbits used in the OD process, or uncoupled attitude control system thrust perhaps for gyro desaturation or star calibrations. ${ }^{3}$ There are also numerous sets of orbits where the change in altitude is of order $10 \mathrm{~m}$ and the change is persistent from one orbit to the next. These are likely orbits that are only perturbed by non-central gravitational forces and solar pressure. Due to the long Venusian day and the nearly spherical gravity field, these orbits are approximately two body ellipses. These altitude changes are so small compared to the density scale heights, that for a particular pair of orbits between corridor control maneuvers, the

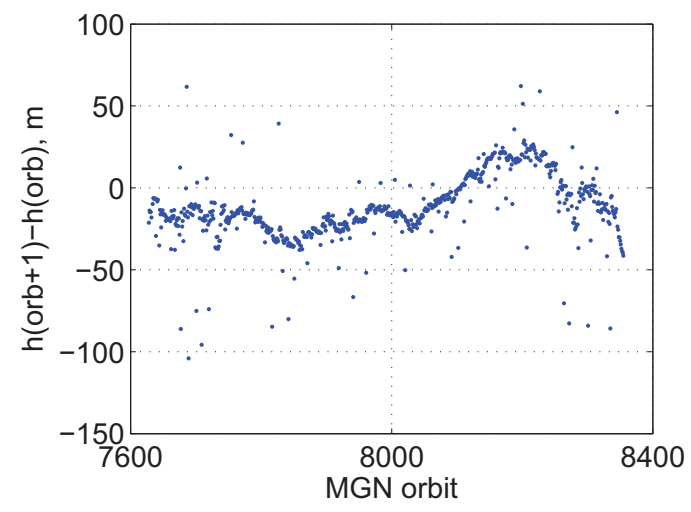

Figure 5. MGN periapsis to periapsis altitude change excluding corridor control maneuvers change in density is essentially orbit to orbit variability over one orbital period.

Figure 6 shows some results from a study designed to determine the orbit to orbit variability by comparing atmospheric density between orbits that have a small periapsis altitude difference. The upper left panel was obtained by selecting, from all the MGN orbits, those with successive periapsis altitude that were within 50m of each other. Over the 695 orbit pairs that satisfied this criterion, the $1 \sigma$ deviation was $5.2 \%$ in the ratio of density at periapsis $(n+1)$ to the density at periapsis $\mathrm{n}$. The variability is clearly higher for the first 200 orbits when the LST is within one hour of noon. Here the $1 \sigma$ variability is $7.8 \%$. The SZA during this time ranges from $9^{\circ}$ to $18^{\circ}$. One model of Venusian thermospheric circulation ${ }^{9}$ suggest an upwelling at the sub solar point $(\mathrm{SZA}=0)$, transport through the upper thermosphere, and subsidence at the anti-sub solar point $\left(\mathrm{SZA}=180^{\circ}\right)$. Even though this global scale flow is thought to be stable, the upwelling might produce local, large scale eddies that could contribute to the increased orbit to orbit variability for SZA near $0^{\circ}$ and $180^{\circ}$.
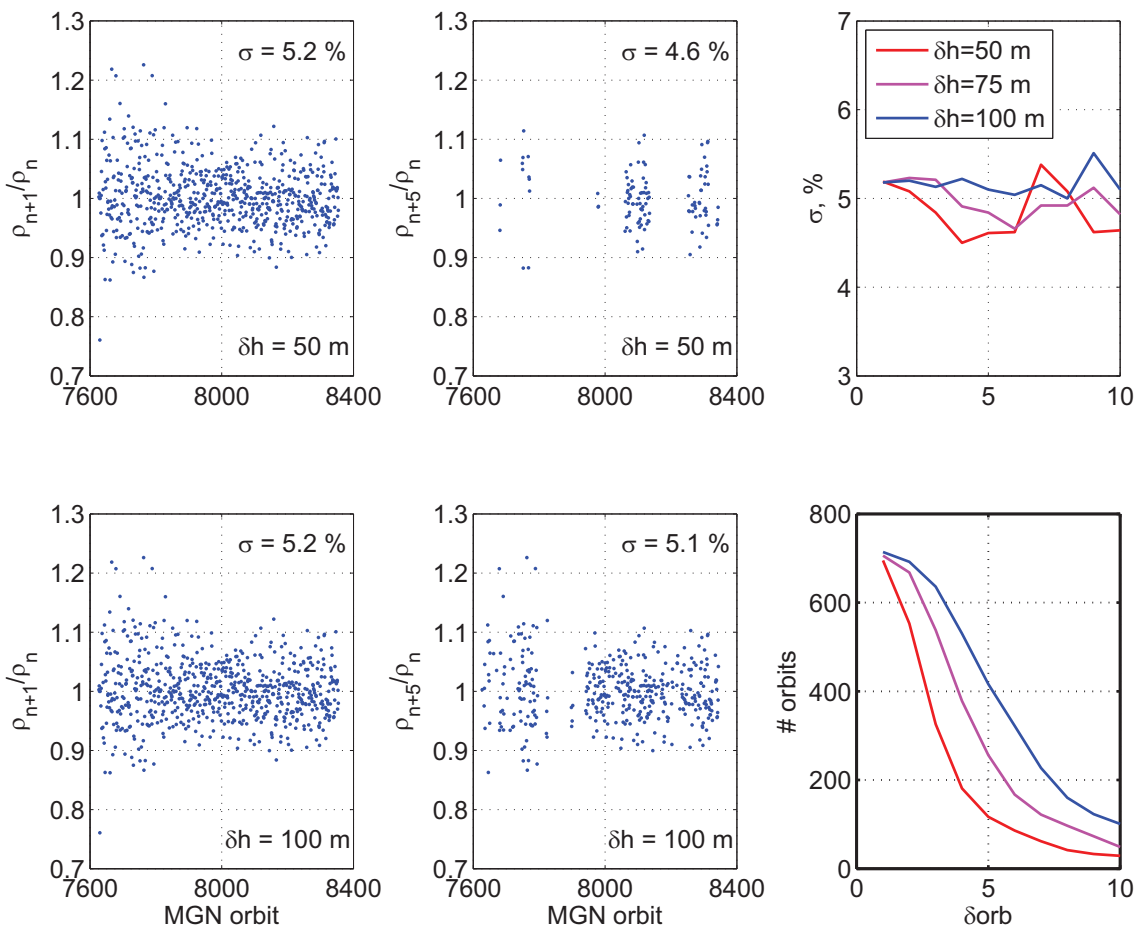

Figure 6. MGN orbit to orbit variability for adjacent orbits within a specified maximum periapsis altitude change. 
If instead of looking at adjacent orbits, the fifth orbit $(n+5)$ after the current orbit $(n)$ is used to determine the variability, the second column is obtained. Here, only 117 orbits meet the criterion, namely those where periapsis altitude is changing more slowly with time. The variability is still around 5\%. The first two lower panels show the results when the altitude difference range is increased to $100 \mathrm{~m}$. Of course more orbits remain when the $(\mathrm{n}+5)$ situation is studied, but variability still remains around 5\%. The last panel in the first row shows the $1 \sigma$ variability for 3 altitude corridors and orbit shifts from 1 to 10 orbits. The lower curve provides the number of orbit pairs for all cases. After a 10 orbit shift, the number of orbits declines to the point where the statistics become questionable. These results suggest that even in the late afternoon the Venusian atmosphere, at main phase aerobraking altitudes, is changing less than $10 \%$ over time scales as long as 7 to 10 hours. But, it must be remembered that these results are from a single mission during the day and with periapsis near the equator.

As seen in Figure 1 there are cycle 4, 5 and 6 data below an altitude to $170 \mathrm{~km}$. This particular set of data included density uncertainties so that data quality could be evaluated before analysis. The night time data are contaminated by orbit determination errors that are larger than the natural variability, but there is a short interval near the end of cycle 4 that can provide some viable results in the morning hours. These data were divided into 1 hour LST intervals and plotted in Figure 7. Only data with relative OD errors less than $10 \%$ are included. Linear trends with LST were removed from the data and the resulting relative errors varied from $7.2 \%$ to $16 \%$. There are clearly some short term trends left in the residuals during some of the one hour intervals, but there are some orbit to orbit changes that are of the order of the standard deviations. To maintain a level of conservatism, no attempt to remove these trends was attempted.
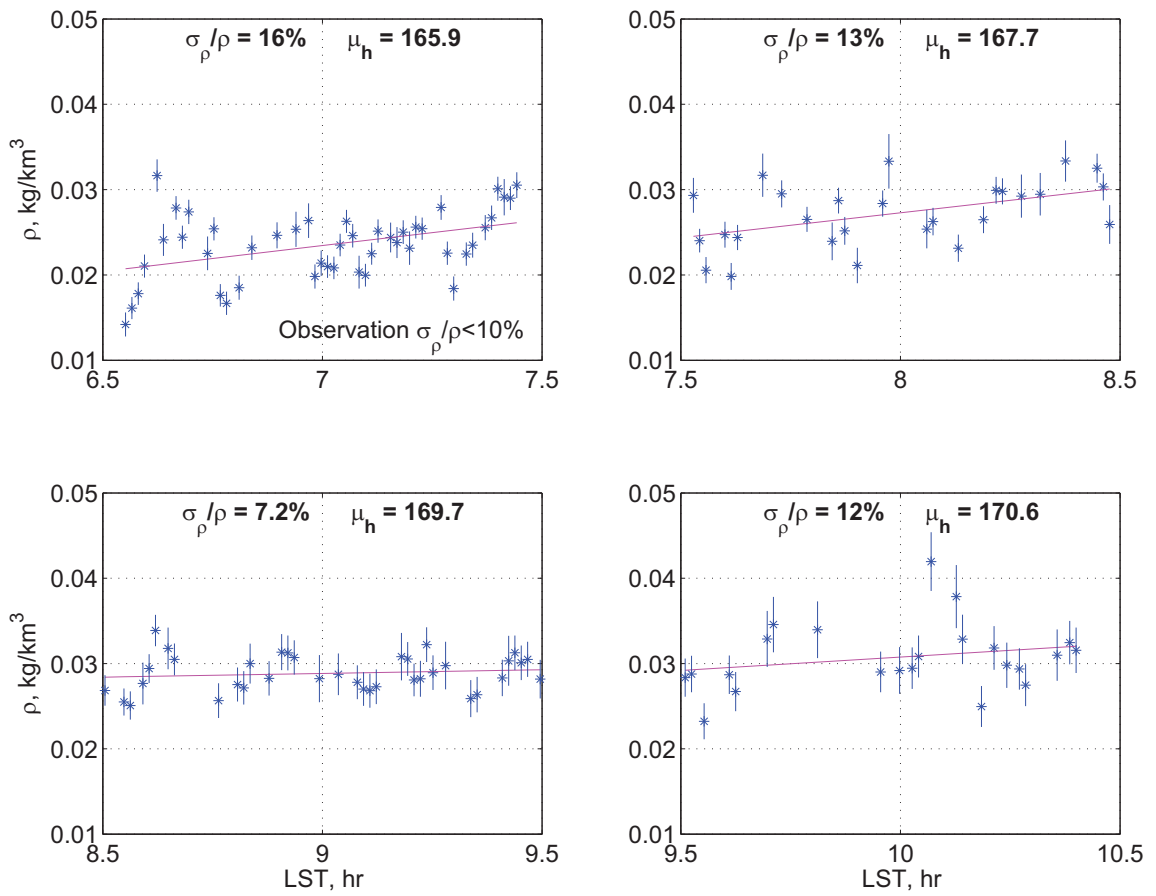

Figure 7. MGN Cycle 4 variability near an altitude of $170 \mathrm{~km}$.

\section{Pioneer Venus Orbiter Low Orbit Variability}

As seen in Figure 3, there are no MGN aerobraking orbits during the night. Like MGN, Figure 1 shows that there are numerous PVO orbits at night and some of the periapsis altitudes are just above the expected aerobraking altitude.

The method used ${ }^{3}$ for determining density at periapsis was to actually solve for density at the reference altitude of $140 \mathrm{~km}$ and use a VIRA like density scale height model to map density to periapsis altitude. In a manner similar to what was done for MGN, orbits within LST intervals of 2 hours are used to determine density models and deviations from these models are considered the natural variability. Throughout the PVO mission the orbital period was held very near 24 hours to provide convenient communication opportunities. With such a large orbital period and eccentricity the solar gravitational perturbations produce orbit to orbit periapsis altitude changes of up to $7 \mathrm{~km}$. Actually 
aerobraking with orbital periods this large will likely require corridor control maneuvers every orbit. Autonomous aerobraking would be very beneficial in this case. ${ }^{10}$

Figure 8 shows the variation of density at periapsis with LST for both years of "low" altitude orbits. During the daytime, the relationship between altitude and density are as expected with what appears to be a linear relation between $\log \rho$ and altitude as seen by comparing upper and lower panels. Before sunrise the expected relationship is still maintained but with what appears to be increased orbit to orbit variability. After sunset, there is no apparent relationship between altitude and density as the variability is large enough to mask any such trend. This is the area of most interest, as these orbits almost reach down to $140 \mathrm{~km}$ altitude.
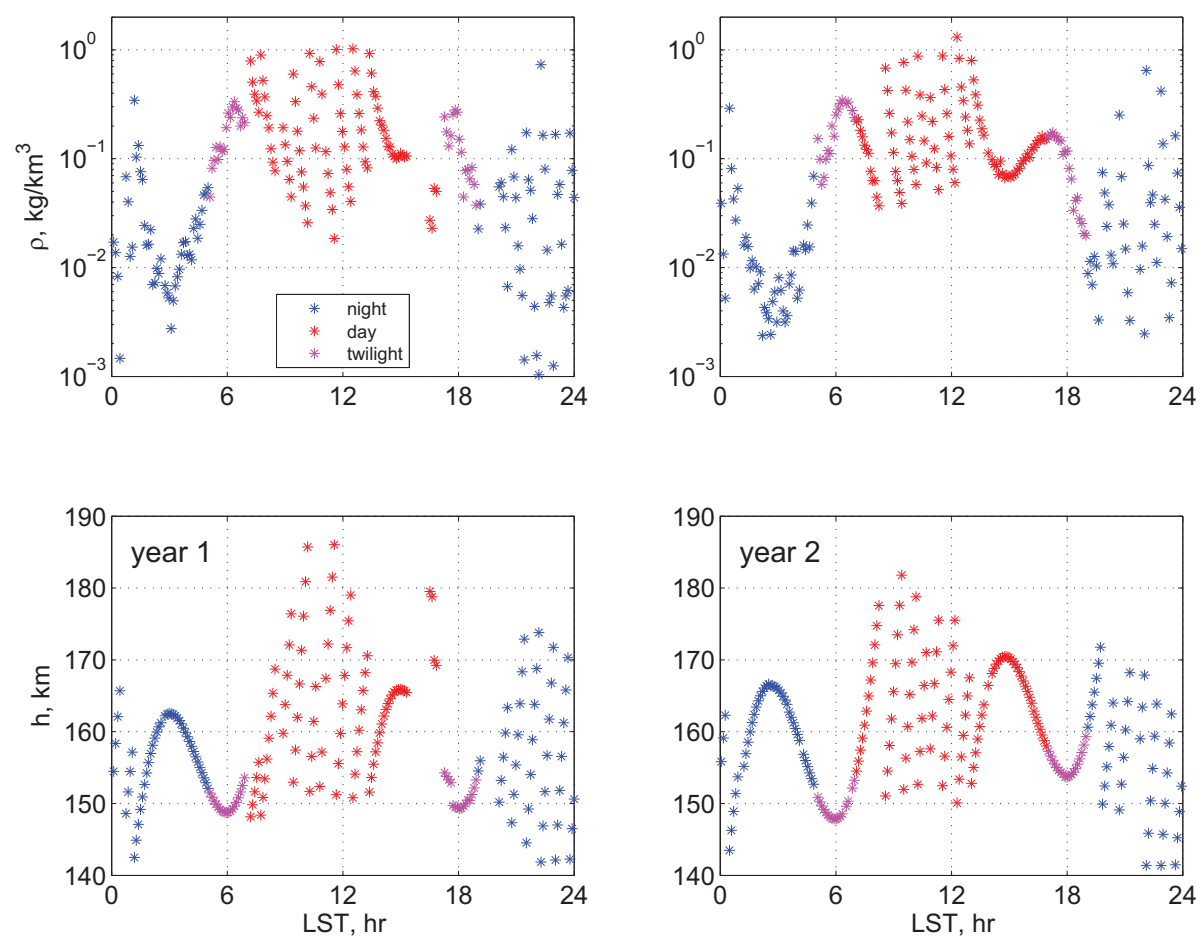

Figure 8. PVO periapsis altitude and density vs. LST for the two years of low altitude orbits. Orbits are identifies as 'day' for LST between 0700 and 1700 hrs., 'night' for LST between 1900 to 0500 hrs. and 'twilight' otherwise.

The day was divided into 2 hour intervals in LST and all data within each interval were used to fit an exponential model across the altitude range. Only intervals with data spanning $10 \mathrm{~km}$ of altitude were considered. The ignored LST intervals correspond to the times when the solar gravitational perturbations to periapsis altitude are the smallest, i.e. LST approximately 0300, 0600, 1500 and 1800 hours. Results for the first and second year are shown in Figure 9 and Figure 10 respectively. The LST range and the number of orbits in the least square fit are shown in the lower left of each panel, the resulting density scale height is also provided and the model is the red line in each panel. The value of $\sigma_{\rho}$ is the standard deviation of $\log \rho$ from the model in percent. For small values of $\sigma$ this is approximately the fractional deviation in density, but for larger values one must consider the non-linear effects. For example, $\sigma=200 \%$ provides an error bound of $\mathrm{e}^{2}$ or a factor of 7.4 density difference. The values of density at the reference altitude are also provided ${ }^{3}$ and the standard deviation for these estimates from the mean value over the same LST intervals are provided as $\sigma_{\rho}$. The values are not identical because the density scale height varies with altitude. The peak standard deviations are between $40 \%$ and $50 \%$ and peak between sunset and midnight. There is generally a decrease through the rest of the night and a dramatic drop with sunrise. Minimum variations of around 10\% occur near midday. During the day, some curvature can be seen in the density profiles likely due to temperature increasing and/or mean molecular weight decreasing with altitude. No attempt has been made to include these small effects in the standard deviations as this leads to slight overestimates of variability, i.e. conservative from an $\mathrm{AB}$ viewpoint. 

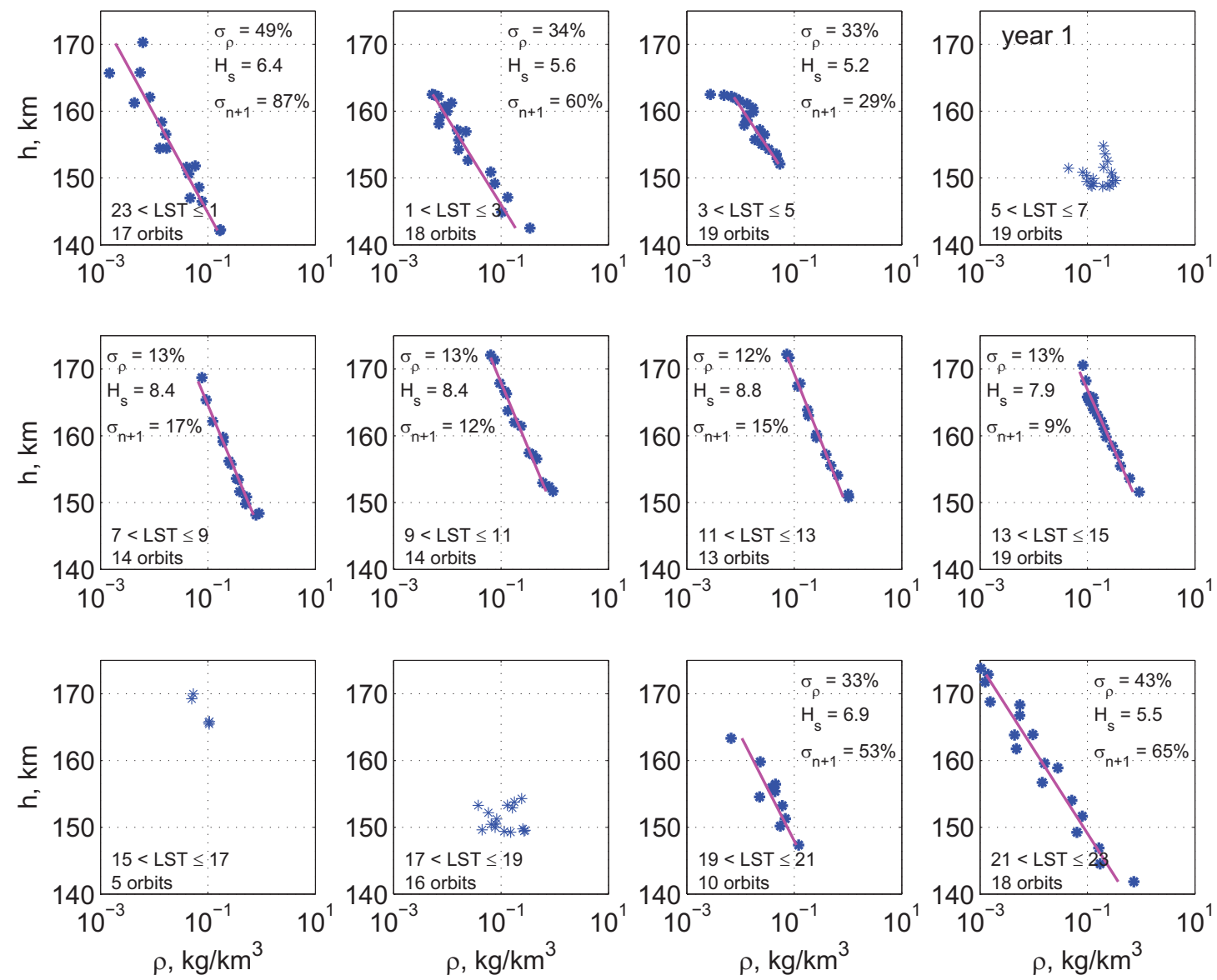

Figure 9. PVO year 1 periapsis density profiles for 2 hour LST intervals. Data fit with constant scale height model. RMS residuals $\left(\sigma_{\rho}\right)$ and scale height given along with number of orbits in the solution. RMS residual at reference altitude of given by $\sigma_{\rho}$. 

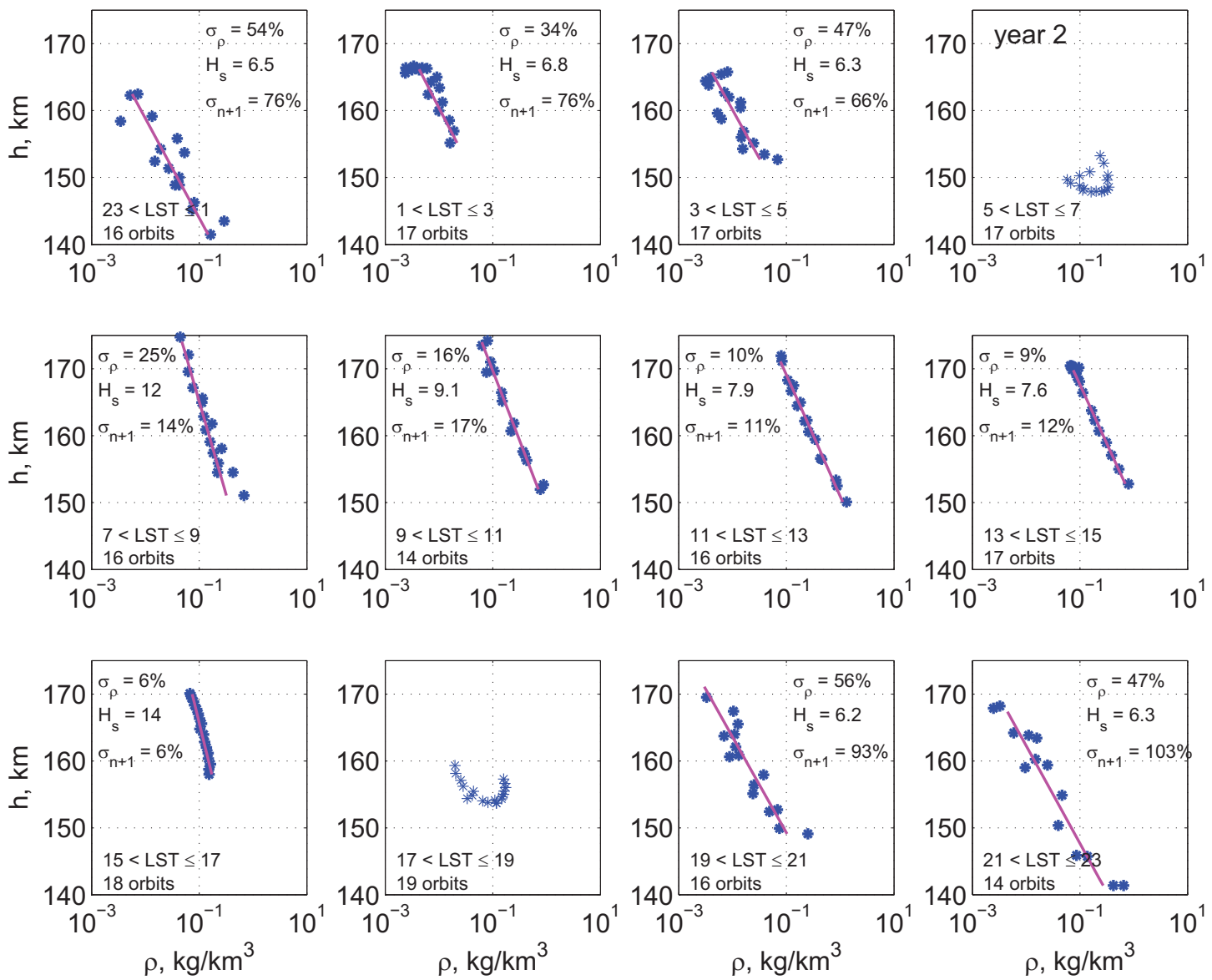

Figure 10. PVO year 2 periapsis density profiles for 2 hour LST intervals. Data fit with constant
scale height model. RMS residuals $\left(\sigma_{\rho}\right)$ and scale height given along with number of orbits in
the solution. RMS residual at reference altitude of given by $\sigma_{\rho^{*}}$

As was the case for MGN, there are sets of adjacent orbits where the altitude changes a small fraction of the density scale height. From Figure 8 these occur around LST=3, 6, 15 and $18 \mathrm{hrs}$. It is of interest to see if density variability at a fixed altitude can be quantified from these orbits. Figure 11 provides the results of this study. Each panel shows a point for each density-altitude pair selected for study. The altitude range was limited to about $0.5 \mathrm{~km}$. At LST $=3$ and 15 hrs, the standard deviations are consistent with those shown in Figure 9 and Figure 10. At LST $=6$ and $18 \mathrm{hrs}$, no standard deviations are provided in Figure 9 and Figure 10 because the data did not represent a log density model. It is seen that in the vicinity of the terminator, $1 \sigma$ deviations range between $30 \%$ and $50 \%$.

For the subset of these data, taken in the vicinity of the terminator, there is a strong suggestion that the density variation is highly correlated with the LST. The simple model with $\log (\rho)$ being linear in altitude is expanded to include a term linear in LST. The model takes the form

$$
\log (\rho)=a+b\left(h-h_{o}\right)+c\left(L S T-L S T_{o}\right)
$$

where $\mathrm{a}, \mathrm{b}$ and $\mathrm{c}$ are determined by a least squares fit to the data set and $\mathrm{h}_{\mathrm{o}}$ and $\mathrm{LST}_{\mathrm{o}}$ are the values at the minimum altitude of the data set. The coefficients represent the $\log \left(\rho_{\mathrm{o}}\right),-1 / \mathrm{H}_{\mathrm{s}}$, and the LST gradient of $\log (\rho)$ at $\mathrm{h}_{\mathrm{o}}$. Extrapolation, with this model, beyond the data set can quickly lead to unrealistic densities. Nevertheless, the model is adequate for the purposed here. Figure 12 provides a summary of this study. Data about 1 hours on either side of sunrise and sunset were selected. The points are the density data and the line is the model using Equation (3). Comparing these $1 \sigma$ values with the corresponding times in Figure 11, shows that the sunrise variation can be reduced to one half and the sunset variation can be reduced to two thirds by using a LST dependent model. It was common practice during Mars AB operations to include similar latitudinal gradients in modeling the atmosphere. ${ }^{11}$ 



